\title{
Author Index Volume 33 (2013)
}

The issue number is given in front of the pagination

Abbruzzese, G., see Frazzitta, G. (2) 299-303

Abdul-sattar, A.B., and T. Godab, Predictors of functional outcome in Saudi Arabian patients with stroke after inpatient rehabilitation (2) 209216

Abián-Vicén, J., see Bravo-Esteban, E. (4) 531-543

Abidi, N.S., see Dodakian, L. (1) 13-24

Acampa, M., see Cioncoloni, D. (2) 217-223

Achiron, A., see Kalron, A. (3) 423-430

Aguirrezabal, A., E. Duarte, N. Rueda, C. Cervantes, E. Marco and F. Escalada, Effects of information and training provision in satisfaction of patients and carers in stroke rehabilitation (4) 639-647

Akbari, S., P.D. Lyden, M. Kamali and M.A. Fahimi, Correlations among impairment, daily activities and thinking operations after stroke (1) 153-160

Albu, S., see Bravo-Esteban, E. (4) 531-543

Alguacil-Diego, I.M., see Gutiérrez, R.O. (4) 545-554 Alguacil-Diego, I.M., see Luna-Oliva, L. (4) 513-521

Alimović, S., A. Katušić and V. Mejaški-Bošnjak, Visual stimulations' critical period in infants with perinatal brain damage (2) 251-255

Almeida, I., see Dores, A.R. (4) 505-512

Altenburger, P., see Ladenheim, B. (1) 25-31

Andrich, J., see Müller, T. (4) 649-655

Andringa, A.S., I.G.L. van de Port and J.-W.G. Meijer, Tolerance and effectiveness of a new dynamic hand-wrist orthosis in chronic stroke patients (2) 225-231

Arango-Lasprilla, J.C., see Stevens, L.F. (2) 273-283

Asch, P.V., see Feys, P. (3) 439-448

Asenbaum-Nan, S., see Salhofer-Polanyi, S. (2) 285292

Avedissian, M., see Cortes, M. (1) 57-65

Baek, S.O., O.L. Kim, S.H. Kim, M.S. Kim, S.M. Son, Y.W. Cho, W.M. Byun and S.H. Jang, Relation between cingulum injury and cognition in chronic patients with traumatic brain injury; diffusion tensor tractography study (3) 465-471

Baert, I., see Feys, P. (3) 439-448

Bagur-Calafat, C., see Cabanas-Valdés, R. (4) 575-592
Barbosa, F., see Dores, A.R. (4) 505-512

Barker, K.D.D., see Pellicane, A.J. (3) 367-376

Barman, A., R. Bhide, A. Viswanathan, J. George, R. Thomas and G. Tharion, Gorham's disease of the spine (1) 121-126

Barton, J.E., see Forrester, L.W. (1) 85-97

Basaglia, N., see Straudi, S. (4) 555-563

Battaglia, M.A., see Brichetto, G. (1) 107-112

Batur-Caglayan, H.Z., see Bayraktar, D. (3) 431-437

Bayraktar, D., A. Guclu-Gunduz, G. Yazici, J. Lambeck, H.Z. Batur-Caglayan, C. Irkec and B. Nazliel, Effects of Ai-Chi on balance, functional mobility, strength and fatigue in patients with multiple sclerosis: A pilot study (3) 431-437

Bayraktar, D., see Citaker, S. (2) 293-298

Benedetti, M.G., see Straudi, S. (4) 555-563

Bernarding, J., see Wang, J. (4) 593-603

Bertotti, G., see Frazzitta, G. (2) 299-303

Bhide, R., see Barman, A. (1) 121-126

Bigazzi, S., see Mazzoleni, S. (1) 33-39

Birbaumer, N., see Broetz, D. (3) 377-384

Bishop, L., and J. Stein, Three upper limb robotic devices for stroke rehabilitation: A review and clinical perspective (1) 3-11

Block, C., and L. Cianfrini, Neuropsychological and neuroanatomical sequelae of chronic nonmalignant pain and opioid analgesia (2) 343-366

Bockbrader, M., see Worthen-Chaudhari, L. (3) 481490

Boveri, N., see Frazzitta, G. (2) 299-303

Bravo-Esteban, E., J. Taylor, J. Abián-Vicén, S. Albu, C. Simón-Martínez, D. Torricelli and J. Gómez-Soriano, Impact of specific symptoms of spasticity on voluntary lower limb muscle function, gait and daily activities during subacute and chronic spinal cord injury (4) 531-543

Brichetto, G., S. Rinaldi, P. Spallarossa, M.A. Battaglia and M.L. Lopes de Carvalho, Efficacy of physical therapy in multiple sclerosis as measured with the modified fatigue impact scale and ambulation index: A retrospective study (1) 107112 
Broetz, D., and N. Birbaumer, Behavioral physiotherapy in post stroke rehabilitation (3) 377-384

Brogårdh, C., see Flansbjer, U.-B. (3) 457-464

Brogårdh, C., U.-B. Flansbjer, C. Espelund and J. Lexell, Relationship between self-reported walking ability and objectively assessed gait performance in persons with late effects of polio (1) $127-132$

Bruce, M.K., see Worthen-Chaudhari, L. (3) 481-490

Brunetti, M., see Wang, J. (4) 593-603

Bugdayci, D., see Paker, N. (2) 323-328

Bugdayci, D., see Paker, N. (2) 337-341

Bunno, Y., see Suzuki, T. (1) 171-176

Byun, W.M., see Baek, S.O. (3) 465-471

Cabanas-Valdés, R., G.U. Cuchi and C. Bagur-Calafat, Trunk training exercises approaches for improving trunk performance and functional sitting balance in patients with stroke: A systematic review (4) 575-592

Caldas, A.C., see Dores, A.R. (4) 505-512

Cano-de la Cuerda, R., see Luna-Oliva, L. (4) 513-521

Cardinal, R., see Ladenheim, B. (1) 25-31

Carrico, C., see Chelette, K.C. (3) 385-389

Carrozza, M.C., see Mazzoleni, S. (1) 33-39

Castelo-Branco, M., see Dores, A.R. (4) 505-512

Celik, B., see Paker, N. (2) 337-341

Cervantes, C., see Aguirrezabal, A. (4) 639-647

Chelette, K.C., C. Carrico, L. Nichols and L. Sawaki, Long-term cortical reorganization following stroke in a single subject with severe motor impairment (3) 385-389

Chelette, K.C., see Danzl, M.M. (1) 67-76

Cho, G.M., see Gorgey, A.S. (1) 161-170

Cho, Y.W., see Baek, S.O. (3) 465-471

Cianfrini, L., see Block, C. (2) 343-366

Cioncoloni, D., G. Martini, P. Piu, S. Taddei, M. Acampa, F. Guideri, R. Tassi and R. Mazzocchio, Predictors of long-term recovery in complex activities of daily living before discharge from the stroke unit (2) 217-223

Citaker, S., A. Guclu-Gunduz, G. Yazici, D. Bayraktar, B. Nazliel and C. Irkec, Relationship between lower extremity isometric muscle strength and standing balance in patients with multiple sclerosis (2) 293-298

Colombo, R., I. Sterpi, A. Mazzone, C. Delconte and F. Pisano, Robot-aided neurorehabilitation in sub-acute and chronic stroke: Does spontaneous recovery have a limited impact on outcome? (4) 621-629
Corbett, D.B., K.S. Peer and A.L. Ridgel, Biomechanical muscle stimulation and active-assisted cycling improves active range of motion in individuals with Parkinson's disease (2) 313-322

Cortes, M., J. Elder, A. Rykman, L. Murray, M. Avedissian, A. Stampas, G.W. Thickbroom, A. Pascual-Leone, H.I. Krebs, J. Valls-Sole and D.J. Edwards, Improved motor performance in chronic spinal cord injury following upper-limb robotic training (1) 57-65

Cramer, S.C., see Dodakian, L. (1) 13-24

Cuchi, G.U., see Cabanas-Valdés, R. (4) 575-592

Cup, E.H.C., see Ijspeert, J. (4) 657-665

Danzl, M.M., K.C. Chelette, K. Lee, D. Lykins and L. Sawaki, Brain stimulation paired with novel locomotor training with a robotic gait orthosis in chronic stroke: A feasibility study (1) 67-76

Dario, P., see Mazzoleni, S. (1) 33-39

de la Cuerda, R.C., see Gutiérrez, R.O. (4) 545-554

de Sousa, L., see Dores, A.R. (4) 505-512

del Carmen Martínez Culebras, M., see Luna-Oliva, L. (4) $513-521$

del Río, F.G., see Gutiérrez, R.O. (4) 545-554

Delconte, C., see Colombo, R. (4) 621-629

Dendale, P., see Hansen, D. (1) 139-146

Depolo Passalacqua, M., see Guillén-Solà, A. (4) 631638

Dierks, T., see Ladenheim, B. (1) 25-31

Diermayr, G., see Salhofer-Polanyi, S. (2) 285-292

Dipietro, L., see Giacobbe, V. (1) 49-56

DiPiro, N.D., see Saunders, L.L. (3) 449-456

Dodakian, L., K.G. Sharp, J., See, N.S. Abidi, K. Mai, B.W. Fling, V.H. Le and S.C. Cramer, Targeted engagement of a dorsal premotor circuit in the treatment of post-stroke paresis (1) 13-24

Dohle, C., see Wang, J. (4) 593-603

Dolbow, D.R., see Gorgey, A.S. (1) 161-170

Donaire Mejías, M.F., see Guillén-Solà, A. (4) 631-638

Dores, A.R., I. Almeida, F. Barbosa, M. CasteloBranco, L. Monteiro, M. Reis, L. de Sousa and A.C. Caldas, Effects of emotional valence and three-dimensionality of visual stimuli on brain activation: An fMRI study (4) 505-512

Dragin, A., see Miljković, N. (3) 491-501

Duarte, E., see Aguirrezabal, A. (4) 639-647

Duarte, E., see Guillén-Solà, A. (4) 631-638

Duret, C., and E. Hutin, Effects of prolonged robotassisted training on upper limb motor recovery in subacute stroke (1) 41-48

Dvir, Z., see Kalron, A. (3) 423-430 
Edwards, D.J., see Cortes, M. (1) 57-65

Edwards, D.J., see Giacobbe, V. (1) 49-56

Eijnde, B.O., see Hansen, D. (1) 139-146

Ekoja, E., see Saunders, L.L. (3) 449-456

Elder, J., see Cortes, M. (1) 57-65

Ellis, C., K.L. Focht and A.L. Grubaugh, Perceptions of stroke recovery: An exclusion of communication and cognition (2) 233-239

Escalada, F., see Aguirrezabal, A. (4) 639-647

Escalada, F., see Guillén-Solà, A. (4) 631-638

Espelund, C., see Brogårdh, C. (1) 127-132

Essmeister, M., see Salhofer-Polanyi, S. (2) 285-292

Fahimi, M.A., see Akbari, S. (1) 153-160

Feys, P., K. Tytgat, D. Gijbels, L.D. Groote, I. Baert and P.V. Asch, Effects of an 1-day education program on physical functioning, activity and quality of life in community living persons with multiple sclerosis (3) 439-448

Fisher, S., see Thrasher, T.A. (1) 133-138

Flansbjer, U.-B., C. Brogårdh and J. Lexell, Muscle strength is only a weak to moderate predictor of gait performance in persons with late effects of polio (3) 457-464

Flansbjer, U.-B., see Brogårdh, C. (1) 127-132

Fling, B.W., see Dodakian, L. (1) 13-24

Focht, K.L., see Ellis, C. (2) 233-239

Forrester, L.W., A. Roy, R.N. Goodman, J. Rietschel, J.E. Barton, H.I. Krebs and R.F. Macko, Clinical application of a modular ankle robot for stroke rehabilitation (1) 85-97

Fortunato, G.J., see San Luis, C.O.V. (2) 201-208

Foti, C., see Straudi, S. (4) 555-563

Franceschini, M., see Mazzoleni, S. (1) 33-39

Frazzitta, G., G. Abbruzzese, G. Bertotti, N. Boveri, G. Pezzoli and R. Maestri, Effectiveness of an intensive rehabilitation treatment on different Parkinson's disease subtypes (2) 299-303

Fregni, F., see Giacobbe, V. (1) 49-56

Fritzsch, C., see Wang, J. (4) 593-603

Gater, D.R., see Gorgey, A.S. (1) 161-170

George, J., see Barman, A. (1) 121-126

Giacobbe, V., H.I. Krebs, B.T. Volpe, A. Pascual-Leone, A. Rykman, G. Zeiarati, F. Fregni, L. Dipietro, G.W. Thickbroom and D.J. Edwards, Transcranial direct current stimulation (tDCS) and robotic practice in chronic stroke: The dimension of timing (1) 49-56

Giacobbi, M., see Merlo, A. (4) 523-530

Giannotti, E., see Merlo, A. (4) 523-530
Gijbels, D., see Feys, P. (3) 439-448

Godab, T., see Abdul-sattar, A.B. (2) 209-216

Goksenoglu, G., see Paker, N. (2) 323-328

Gómez-Soriano, J., see Bravo-Esteban, E. (4) 531-543

González, R.A., see Gutiérrez, R.O. (4) 545-554

Goodman, R.N., see Forrester, L.W. (1) 85-97

Gorgey, A.S., G.M. Cho, D.R. Dolbow and D.R. Gater, Differences in current amplitude evoking leg extension in individuals with spinal cord injury (1) $161-170$

Govil, K., and M.M. Noohu, Effect of EMG biofeedback training of gluteus maximus muscle on gait parameters in incomplete spinal cord injury (1) 147-152

Gregory-Bass, R., see Saunders, L.L. (3) 449-456

Grill, H., see Salhofer-Polanyi, S. (2) 285-292

Groote, L.D., see Feys, P. (3) 439-448

Groothuis, J.T., see Ijspeert, J. (4) 657-665

Grubaugh, A.L., see Ellis, C. (2) 233-239

Guclu-Gunduz, A., see Bayraktar, D. (3) 431-437

Guclu-Gunduz, A., see Citaker, S. (2) 293-298

Guideri, F., see Cioncoloni, D. (2) 217-223

Guillén-Solà, A., E. Marco, J. Martínez-Orfila, M.F. Donaire Mejías, M. Depolo Passalacqua, E. Duarte and F. Escalada, Usefulness of the volume-viscosity swallow test for screening dysphagia in subacute stroke patients in rehabilitation income (4) 631-638

Gurevich, M., see Kalron, A. (3) 423-430

Gutiérrez, R.O., F.G. del Río, R.C. de la Cuerda, I.M. Alguacil-Diego, R.A. González and J.C.M. Page, A telerehabilitation program by virtual realityvideo games improves balance and postural control in multiple sclerosis patients (4) 545-554

Hansen, D., I. Wens, P. Dendale and B.O. Eijnde, Exercise-onset heart rate increase is slowed in multiple sclerosis patients: Does a disturbed cardiac autonomic control affect exercise tolerance? (1) 139-146

Haq, I., see Stegemöller, E.L. (2) 305-312

Harati, A., see Müller, T. (4) 649-655

Haserodt, S., see Worthen-Chaudhari, L. (3) 481-490

Hass, C.J., see Stegemöller, E.L. (2) 305-312

Hesse, S., N. Schattat, J. Mehrholz and C. Werner, Evidence of end-effector based gait machines in gait rehabilitation after CNS lesion (1) 77-84

Hirano, S., see Tanabe, S. (1) 99-106

Hubbard, R., see Stevens, L.F. (2) 273-283

Hutin, E., see Duret, C. (1) 41-48

Hwynn, N., see Stegemöller, E.L. (2) 305-312

Hyun-Nam, S., see Lee, N.K. (1) 177-183 
Ijspeert, J., R.M.J. Janssen, A. Murgia, M.F. Pisters, E.H.C. Cup, J.T. Groothuis and N. van Alfen, Efficacy of a combined physical and occupational therapy intervention in patients with subacute neuralgic amyotrophy: A pilot study (4) 657-665

Irkec, C., see Bayraktar, D. (3) 431-437

Irkec, C., see Citaker, S. (2) 293-298

Jamil, A., see Kholod, H. (1) 113-119

Jang, S.H., Motor recovery by improvement of limbkinetic apraxia in a chronic stroke patient (2) 195200

Jang, S.H., see Baek, S.O. (3) 465-471

Jang, S.H., see Seo, J.P. (2) 257-261

Janssen, R.M.J., see Ijspeert, J. (4) 657-665

Jove, I.G.E., see Stevens, L.F. (2) 273-283

Jughters, A., see Lisa, L.P. (4) 611-620

Kalron, A., Z. Dvir, M. Gurevich and A. Achiron, Do motor impairments detected on onset of multiple sclerosis suggest an early second attack? A prospective study (3) 423-430

Kamali, M., see Akbari, S. (1) 153-160

Kammersgaard, L.P., M. Linnemann and M. Tibæk, Hydrocephalus following severe traumatic brain injury in adults. Incidence, timing, and clinical predictors during rehabilitation (3) 473-480

Kang, K.W., see Lee, N.K. (1) 177-183

Kang, M.-H., see Lee, D.-K. (4) 605-610

Katušić, A., see Alimović, S. (2) 251-255

Katz-Leurer, M., see Kholod, H. (1) 113-119

Kerckhofs, E., see Lisa, L.P. (4) 611-620

Kesiktas, N., see Paker, N. (2) 323-328

Kesiktas, N., see Paker, N. (2) 337-341

Khan, C.M., and P. Oesch, Validity and responsiveness of the German version of the Motor Activity Log for the assessment of self-perceived arm use in hemiplegia after stroke (3) 413-421

Kholod, H., A. Jamil and M. Katz-Leurer, The associations between motor ability, walking activity and heart rate and heart rate variability parameters among children with cerebral palsy and typically developed controls (1) 113-119

Kim, C.-J., see Sung, Y.-H. (3) 407-412

Kim, J.-H., and B.-H. Lee, Action observation training for functional activities after stroke: A pilot randomized controlled trial (4) 565-574

Kim, J.-W., see Lee, D.-K. (4) 605-610

Kim, K., see Lee, N.K. (1) 177-183

Kim, K.-M., see Sung, Y.-H. (3) 407-412

Kim, M.S., see Baek, S.O. (3) 465-471
Kim, O.L., see Baek, S.O. (3) 465-471

Kim, S.H., see Baek, S.O. (3) 465-471

Kim, Y.-G., see Lee, D.-K. (4) 605-610

Konstantinović, L., see Miljković, N. (3) 491-501

Krause, J.S., see Saunders, L.L. (3) 449-456

Krause, T., see Wang, J. (4) 593-603

Krebs, H.I., see Cortes, M. (1) 57-65

Krebs, H.I., see Forrester, L.W. (1) 85-97

Krebs, H.I., see Giacobbe, V. (1) 49-56

Krebs, H.I., see Ladenheim, B. (1) 25-31

Kwon, J.W., see Lee, N.K. (1) 177-183

Kwon, O.S., and S.W. Lee, Effect of continuing repeated passive and active exercises on knee's position senses in patients with hemiplegia (3) 391-397

Ladenheim, B., P. Altenburger, R. Cardinal, L. Monterroso, T. Dierks, J. Mast and H.I. Krebs, The effect of random or sequential presentation of targets during robot-assisted therapy on children (1) 25 31

Lah, S., see Saxton, M.E. (2) 263-271

Lambeck, J., see Bayraktar, D. (3) 431-437

Le, V.H., see Dodakian, L. (1) 13-24

Lee, B.-H., see Kim, J.-H. (4) 565-574

Lee, D.-K., M.-H. Kang, J.-W. Kim, Y.-G. Kim, J.H. Park and J.-S. Oh, Effects of non-paretic arm exercises using a tubing band on abdominal muscle activity in stroke patients (4) 605-610

Lee, K., see Danzl, M.M. (1) 67-76

Lee, N.K., J.W. Kwon, S.M. Son, K.W. Kang, K. Kim and $\mathrm{S}$. Hyun-Nam, The effects of closed and open kinetic chain exercises on lower limb muscle activity and balance in stroke survivors (1) 177-183

Lee, S.W., see Kwon, O.S. (3) 391-397

Leutmezer, F., see Salhofer-Polanyi, S. (2) 285-292

Lexell, J., see Brogårdh, C. (1) 127-132

Lexell, J., see Flansbjer, U.-B. (3) 457-464

Linnemann, M., see Kammersgaard, L.P. (3) 473-480

Lisa, L.P., A. Jughters and E. Kerckhofs, The effectiveness of different treatment modalities for the rehabilitation of unilateral neglect in stroke patients: A systematic review (4) 611-620

Lombardi, B., see Troncati, F. (3) 399-405

Longhi, M., see Merlo, A. (4) 523-530

Lopes de Carvalho, M.L., see Brichetto, G. (1) 107-112 Luna-Oliva, L., R.M. Ortiz-Gutiérrez, R. Cano-de la Cuerda, R.M. Piédrola, I.M. Alguacil-Diego, C. Sánchez-Camarero and M. del Carmen Martínez Culebras, Kinect Xbox 360 as a therapeutic modality for children with cerebral palsy in a 
school environment: A preliminary study (4) 513521

Lyden, P.D., see Akbari, S. (1) 153-160

Lykins, D., see Danzl, M.M. (1) 67-76

Macko, R.F., see Forrester, L.W. (1) 85-97

Maestri, R., see Frazzitta, G. (2) 299-303

Mai, K., see Dodakian, L. (1) 13-24

Malcolm, M.P., see Massie, C.L. (2) 185-193

Manca, M., see Straudi, S. (4) 555-563

Mancini, A., see Merlo, A. (4) 523-530

Marco, E., see Aguirrezabal, A. (4) 639-647

Marco, E., see Guillén-Solà, A. (4) 631-638

Martínez-Orfila, J., see Guillén-Solà, A. (4) 631-638

Martini, G., see Cioncoloni, D. (2) 217-223

Massie, C.L., B.L. Tracy, R.J. Paxton and M.P. Malcolm, Repeated sessions of functional repetitive transcranial magnetic stimulation increases motor cortex excitability and motor control in survivors of stroke (2) 185-193

Mast, J., see Ladenheim, B. (1) 25-31

Mauritz, K.-H., see Wang, J. (4) 593-603

Mazzocchio, R., see Cioncoloni, D. (2) 217-223

Mazzoleni, S., P. Sale, M. Franceschini, S. Bigazzi, M.C. Carrozza, P. Dario and F. Posteraro, Effects of proximal and distal robot-assisted upper limb rehabilitation on chronic stroke recovery (1) 33-39

Mazzoli, D., see Merlo, A. (4) 523-530

Mazzone, A., see Colombo, R. (4) 621-629

McCullough, L.D., see San Luis, C.O.V. (2) 201-208

Mehrholz, J., see Hesse, S. (1) 77-84

Meijer, J.-W.G., see Andringa, A.S. (2) 225-231

Mejaški-Bošnjak, V., see Alimović, S. (2) 251-255

Merlo, A., M. Longhi, E. Giannotti, P. Prati, M. Giacobbi, E. Ruscelli, A. Mancini, M. Ottaviani, L. Montanari and D. Mazzoli, Upper limb evaluation with robotic exoskeleton. Normative values for indices of accuracy, speed and smoothness (4) 523-530

Midik, M., see Paker, N. (2) 337-341

Miljković, N., I. Milovanović, A. Dragin, L. Konstantinović and D.B. Popović, Muscle synergies with Walkaround $\AA$ postural support vs. "cane/therapist" assistance (3) 491-501

Millis, S.R., see Pellicane, A.J. (3) 367-376

Milovanović, I., see Miljković, N. (3) 491-501

Montanari, L., see Merlo, A. (4) 523-530

Monteiro, L., see Dores, A.R. (4) 505-512

Monterroso, L., see Ladenheim, B. (1) 25-31

Müller, T., C. Saft, J. Andrich and A. Harati, Diadochokinetic movements differ between patients with Huntington's disease and controls (4) 649-655

Murgia, A., see Ijspeert, J. (4) 657-665

Murray, L., see Cortes, M. (1) 57-65

Myftari, T., see Troncati, F. (3) 399-405

Mysiw, W.J., see Worthen-Chaudhari, L. (3) 481-490

Nazliel, B., see Bayraktar, D. (3) 431-437

Nazliel, B., see Citaker, S. (2) 293-298

Nichols, L., see Chelette, K.C. (3) 385-389

Noohu, M.M., see Govil, K. (1) 147-152

O’Neil, R.L., R.L. Skeel and K.I. Ustinova, Cognitive ability predicts motor learning on a virtual reality game in patients with TBI (4) 667-680

Oesch, P., see Khan, C.M. (3) 413-421

Oh, J.-S., see Lee, D.-K. (4) 605-610

Okun, M.S., see Stegemöller, E.L. (2) 305-312

Ollenschleger, M.D., see San Luis, C.O.V. (2) 201-208

Onigata, C., see Suzuki, T. (1) 171-176

Ortiz-Gutiérrez, R.M., see Luna-Oliva, L. (4) 513-521

Oswald, M.C., see Pellicane, A.J. (3) 367-376

Ottaviani, M., see Merlo, A. (4) 523-530

Owolabi, M.O., Psychometric properties of the German version of the health-related quality of life in stroke patients (HRQOLISP) instrument (2) 241250

Paci, M., see Troncati, F. (3) 399-405

Page, J.C.M., see Gutiérrez, R.O. (4) 545-554

Paker, N., D. Bugdayci, G. Goksenoglu, A. Sen and N. Kesiktas, Effects of robotic treadmill training on functional mobility, walking capacity, motor symptoms and quality of life in ambulatory patients with Parkinson's disease: A preliminary prospective longitudinal study (2) 323-328

Paker, N., D. Bugdayci, M. Midik, B. Celik and N. Kesiktas, Reliability of the Turkish version of the hospital anxiety and depression scale in the people with traumatic spinal cord injury (2) 337-341

Park, J.-H., see Lee, D.-K. (4) 605-610

Pascual-Leone, A., see Cortes, M. (1) 57-65

Pascual-Leone, A., see Giacobbe, V. (1) 49-56

Paxton, R.J., see Massie, C.L. (2) 185-193

Peer, K.S., see Corbett, D.B. (2) 313-322

Pellicane, A.J., S.R. Millis, K.D.D. Barker, K.E. Temme, A. Sayyad, M.C. Oswald and E.J. Roth, The effect of protein and calorie intake on prealbumin, complications, length of stay, and function in the acute rehabilitation inpatient with stroke (3) 367-376 
Perrin, P.B., see Stevens, L.F. (2) 273-283

Pezzoli, G., see Frazzitta, G. (2) 299-303

Piédrola, R.M., see Luna-Oliva, L. (4) 513-521

Pisano, F., see Colombo, R. (4) 621-629

Pisters, M.F., see Ijspeert, J. (4) 657-665

Piu, P., see Cioncoloni, D. (2) 217-223

Popović, D.B., see Miljković, N. (3) 491-501

Posteraro, F., see Mazzoleni, S. (1) 33-39

Prati, P., see Merlo, A. (4) 523-530

Reis, M., see Dores, A.R. (4) 505-512

Reitz, A., Lower urinary tract dysfunction in critical illness polyneuropathy (2) 329-336

Ridgel, A.L., see Corbett, D.B. (2) 313-322

Rietschel, J., see Forrester, L.W. (1) 85-97

Rinaldi, S., see Brichetto, G. (1) 107-112

Roth, E.J., see Pellicane, A.J. (3) 367-376

Roy, A., see Forrester, L.W. (1) 85-97

Rueda, N., see Aguirrezabal, A. (4) 639-647

Ruscelli, E., see Merlo, A. (4) 523-530

Rykman, A., see Cortes, M. (1) 57-65

Rykman, A., see Giacobbe, V. (1) 49-56

Saft, C., see Müller, T. (4) 649-655

Saitoh, E., see Tanabe, S. (1) 99-106

Sale, P., see Mazzoleni, S. (1) 33-39

Salhofer-Polanyi, S., J. Windt, H. Sumper, H. Grill, M. Essmeister, G. Diermayr, K. Zebenholzer, F. Leutmezer, G. Zulehner, K. Vass and S. AsenbaumNan, Benefits of inpatient multidisciplinary rehabilitation in multiple sclerosis (2) 285-292

San Luis, C.O.V., I. Staff, M.D. Ollenschleger, G.J. Fortunato and L.D. McCullough, Percutaneous endoscopic gastrostomy tube placement in left versus right middle cerebral artery stroke: Effects of laterality (2) 201-208

Sánchez-Camarero, C., see Luna-Oliva, L. (4) 513-521

Saunders, L.L., E. Ekoja, C.S. Whitlock, N.D. DiPiro, R. Gregory-Bass and J.S. Krause, A comparison of health behaviors between African Americans with spinal cord injury and those in the general population (3) 449-456

Sawaki, L., see Chelette, K.C. (3) 385-389

Sawaki, L., see Danzl, M.M. (1) 67-76

Saxton, M.E., S.S. Younan and S. Lah, Social behaviour following severe traumatic brain injury: Contribution of emotion perception deficits (2) 263-271

Sayyad, A., see Pellicane, A.J. (3) 367-376

Schattat, N., see Hesse, S. (1) 77-84

See, J., see Dodakian, L. (1) 13-24
Sen, A., see Paker, N. (2) 323-328

Seo, J.P., and S.H. Jang, Recovery of injured cingulum in a patient with brain injury: Diffusion tensor tractography study (2) 257-261

Sharp, K.G., see Dodakian, L. (1) 13-24

Simón-Martínez, C., see Bravo-Esteban, E. (4) 531-543

Skeel, R.L., see O’Neil, R.L. (4) 667-680

Smith, R., see Worthen-Chaudhari, L. (3) 481-490

Son, S.M., see Baek, S.O. (3) 465-471

Son, S.M., see Lee, N.K. (1) 177-183

Sosa, D.M.D., see Stevens, L.F. (2) 273-283

Spallarossa, P., see Brichetto, G. (1) 107-112

Staff, I., see San Luis, C.O.V. (2) 201-208

Stampas, A., see Cortes, M. (1) 57-65

Stegemöller, E.L., S. Vallabhajosula, I. Haq, N. Hwynn, C.J. Hass and M.S. Okun, Selective use of low frequency stimulation in Parkinson's disease based on absence of tremor (2) 305-312

Stein, J., see Bishop, L. (1) 3-11

Sterpi, I., see Colombo, R. (4) 621-629

Stevens, L.F., P.B. Perrin, R. Hubbard, D.M.D. Sosa, I.G.E. Jove and J.C. Arango-Lasprilla, Using multiple views of family dynamics to predict the mental health of individuals with TBI and their caregivers in Mexico (2) 273-283

Straudi, S., M.G. Benedetti, E. Venturini, M. Manca, C. Foti and N. Basaglia, Does robot-assisted gait training ameliorate gait abnormalities in multiple sclerosis? A pilot randomized-control trial (4) 555-563

Sumper, H., see Salhofer-Polanyi, S. (2) 285-292

Sung, Y.-H., C.-J. Kim, B.-K. Yu and K.-M. Kim, A hippotherapy simulator is effective to shift weight bearing toward the affected side during gait in patients with stroke (3) 407-412

Suzuki, T., Y. Bunno, C. Onigata, M. Tani and S. Uragami, Excitability of spinal neural function during several motor imagery tasks involving isometric opponens pollicis activity (1) 171-176

Swendal, C., see Worthen-Chaudhari, L. (3) 481-490

Taddei, S., see Cioncoloni, D. (2) 217-223

Tanabe, S., S. Hirano and E. Saitoh, Wearable PowerAssist Locomotor (WPAL) for supporting upright walking in persons with paraplegia (1) 99-106

Tani, M., see Suzuki, T. (1) 171-176

Tassi, R., see Cioncoloni, D. (2) 217-223

Taylor, J., see Bravo-Esteban, E. (4) 531-543

Temme, K.E., see Pellicane, A.J. (3) 367-376

Tharion, G., see Barman, A. (1) 121-126

Thickbroom, G.W., see Giacobbe, V. (1) 49-56 
Thickbroom, G.W., see Cortes, M. (1) 57-65

Thomas, R., see Barman, A. (1) 121-126

Thrasher, T.A., J.S. Ward and S. Fisher, Strength and endurance adaptations to functional electrical stimulation leg cycle ergometry in spinal cord injury (1) 133-138

Tibæk, M., see Kammersgaard, L.P. (3) 473-480

Torricelli, D., see Bravo-Esteban, E. (4) 531-543

Tracy, B.L., see Massie, C.L. (2) 185-193

Troncati, F., M. Paci, T. Myftari and B. Lombardi, Extracorporeal Shock Wave Therapy reduces upper limb spasticity and improves motricity in patients with chronic hemiplegia: A case series (3) 399-405

Tytgat, K., see Feys, P. (3) 439-448

Uragami, S., see Suzuki, T. (1) 171-176

Ustinova, K.I., see O’Neil, R.L. (4) 667-680

Vallabhajosula, S., see Stegemöller, E.L. (2) 305-312

Valls-Sole, J., see Cortes, M. (1) 57-65

van Alfen, N., see Ijspeert, J. (4) 657-665

van de Port, I.G.L., see Andringa, A.S. (2) 225-231

Vass, K., see Salhofer-Polanyi, S. (2) 285-292

Venturini, E., see Straudi, S. (4) 555-563

Viswanathan, A., see Barman, A. (1) 121-126

Volpe, B.T., see Giacobbe, V. (1) 49-56
Wang, J., C. Fritzsch, J. Bernarding, T. Krause, K.-H. Mauritz, M. Brunetti and C. Dohle, Cerebral activation evoked by the mirror illusion of the hand in stroke patients compared to normal subjects (4) 593-603

Ward, J.S., see Thrasher, T.A. (1) 133-138

Wens, I., see Hansen, D. (1) 139-146

Werner, C., see Hesse, S. (1) 77-84

Whalen, C.N., see Worthen-Chaudhari, L. (3) 481-490

Whitlock, C.S., see Saunders, L.L. (3) 449-456

Windt, J., see Salhofer-Polanyi, S. (2) 285-292

Worthen-Chaudhari, L., C.N. Whalen, C. Swendal, M. Bockbrader, S. Haserodt, R. Smith, M.K. Bruce and W.J. Mysiw, A feasibility study using interactive graphic art feedback to augment acute rehabilitation neurorehabilitation therapy (3) 481490

Yazici, G., see Bayraktar, D. (3) 431-437

Yazici, G., see Citaker, S. (2) 293-298

Younan, S.S., see Saxton, M.E. (2) 263-271

Yu, B.-K., see Sung, Y.-H. (3) 407-412

Zebenholzer, K., see Salhofer-Polanyi, S. (2) 285-292

Zeiarati, G., see Giacobbe, V. (1) 49-56

Zulehner, G., see Salhofer-Polanyi, S. (2) 285-292 